Commun. math. Phys. 7, 337-348 (1968)

\title{
Statistical Mechanics of Quantum Spin Systems. II*
}

\author{
DereK W. RobINSON \\ Lyman Laboratory of Physics, Harvard University
}

Received December 15, 1967

\begin{abstract}
In the first part of this paper we continue the general analysis of quantum spin systems. It is demonstrated, for a large class of interactions, that timetranslations form a group of automorphisms of the $C^{*}$-algebra $\mathfrak{A}$ of quasi-local observables and that the thermodynamic equilibrium states are invariant under this group. Further it is shown that the equilibrium states possess the Kubo-MartinSchwinger analyticity and boundary condition properties. In the second part of the paper we give a general analysis of states which are invariant under space and time translations and also satisfy the KMS boundary condition. A discussion of these latter conditions and their connection with the decomposition of invariant states into ergodic states is given. Various properties pertinent to this discussion are derived.
\end{abstract}

\section{Introduction}

In a previous paper [1], hereafter referred to as $I$, the general analysis of the statistical mechanics of quantum spin system was begun. The primary purpose of the present paper is to continue this analysis. We focus our attention on the problems involved with the time-development of such a system in equilibrium. Our analysis begins with a proof that time-translations form a one-parameter group of automorphisms of the $C^{*}$-algebra $\mathfrak{A}$ of quasi-local observables. It is then possible to demonstrate that the single phase equilibrium states, whose existence was established in I, are both invariant under time translations and satisfy the KuboMartin-Schwinger analyticity and boundary properties.

Recently HaAg, Hugenholtz, and Winnink [2], have studied the properties of states invariant under time translations satisfying the KMS boundary condition and have derived a number of properties of such states which are independent of spatial structure. In the second half of this paper we give an analysis of some of the implications of local structure and invariance under space translations. The KMS boundary condition plays somewhat the role of a spectrum condition and allows us to derive properties whose analog in relativistic field theory is dependent on the spectrum condition. In particular it is shown that time translations leave the center of the covariance algebra, generated by $\mathfrak{A}$ and the group

* Supported in part by the Office of Naval Research Contract No. Nonr 1866(5). 
of space translations, pointwise invariant. Further one may demonstrate that the decomposition of states into extremal space translation invariant states respect the KMS boundary condition. Finally one finds that this condition gives connections between the spectra of space and time translations. These latter results are valid in a more general framework than that of quantum spin systems.

\section{Basic Notation for Spin Systems}

Consider a $\nu$-dimensional lattice $Z^{\nu}$ and associate with each point $x \in Z^{v}$ a Hilbert space of finite dimension $N$. Further, associate with each finite set $\Lambda \subset Z^{\nu}$ the direct product space $\mathscr{H}_{\Lambda}=\prod_{x \in \Lambda}^{\otimes} \mathscr{H}_{x}$. Define the algebra of (strictly) local observables $\mathfrak{A}(\Lambda)$ corresponding to $\Lambda$ as the algebra $\mathfrak{B}\left(\mathscr{H}_{\Lambda}\right)$ of all bounded operators acting on $\mathscr{H}_{\Lambda} ; \mathfrak{A}(\Lambda)$ is a finite matrix algebra. Now if $\Lambda_{1} \subset \Lambda_{2}$ an operator $A_{1} \in \mathfrak{A}\left(\Lambda_{1}\right)$ may be identified with an operators $A_{2} \in \mathfrak{U}\left(\Lambda_{2}\right)$ by setting $A_{2}=A_{1} \otimes 1_{\Lambda_{2} / \Lambda_{1}}$ where $1_{\Lambda}$ is the identity operator on $\mathscr{H}_{\Lambda}$ and $\Lambda_{2} / \Lambda_{1}$ denotes the complement of $\Lambda_{1}$ in $\Lambda_{2}$. This identification induces a norm preserving mapping $\mathfrak{A}\left(\Lambda_{1}\right) \rightarrow \mathfrak{A}\left(\Lambda_{2}\right)$ of the abstract $C^{*}$-algebras and the isotony relation $\mathfrak{A}\left(\Lambda_{1}\right) \subset \mathfrak{A}\left(\Lambda_{2}\right)$ for $\Lambda_{1} \subset \Lambda_{2}$. Due to this isotony relation the set theoretic union of all $\mathfrak{A}(\Lambda)$, with $\Lambda \subset Z^{\nu}$ finite, is a normed *-algebra and we define the completion of this algebra to be the abstract $C^{*}$-algebra $\mathfrak{A}$ of (quasi-) local observables. Note that because the $\mathfrak{A}(\Lambda)$ are simple algebras and are dense in $\mathfrak{A}$ the algebra $\mathfrak{A}$ is also simple i. e. the algebra $\mathfrak{A}$ has no non-trivial twosided ideals ${ }^{1}$. The group $Z^{v}$ of space tanslations is a subgroup of the automorphism group of $\mathfrak{A}$ whose action we denote by $A \in \mathfrak{U}(\Lambda) \rightarrow \tau_{x} A$ $\in \mathfrak{A}(\Lambda+x)$ for $x \in Z^{v}$. The local subalgebras $\mathfrak{A}(\Lambda)$ satisfy the commutation relations

$$
\left[\mathfrak{A}\left(\Lambda_{1}\right), \mathfrak{A}\left(\Lambda_{2}\right)\right]=0 \text { if } \Lambda_{1} \cap \Lambda_{2}=\emptyset,
$$

and $\mathfrak{A}$ is asymptotically abelian with respect to space translations i.e.

$$
\left\|\left[A, \tau_{x} B\right]\right\| \underset{|x|=\infty}{\longrightarrow} 0, A, B \in \mathfrak{A} \text { and } x \in Z^{\nu} .
$$

We consider the sites of the lattice $Z^{v}$ to be occupied by particles which interact through many body "potentials" $\Phi^{(k)}\left(x_{1}, \ldots x_{k}\right) \in \mathfrak{A}\left(\left\{x_{1}, \ldots x_{k}\right\}\right)$ and define an interaction $\Phi$ as a sequence $\Phi=\left(\Phi^{(k)}\right)_{k \geqq 1}$ of $k$-body "potentials". The interaction $\Phi$ may be regarded as a function from the finite sets $X \subset Z^{v}$ to elements of $\mathfrak{A}$ defined by $\Phi(X)=\Phi^{(k)}\left(x_{1}, \ldots x_{k}\right)$ $\in \mathfrak{R}(X)$ if $X=\left\{x_{1}, \ldots x_{k}\right\}$. We consider only those interactions $\Phi$ which have the following properties

\footnotetext{
1 For a general discussion of such algebras see J. GLrmm [8].
} 
I. $\Phi(X)$ is Hermitian for $X \subset Z^{v}$

and

II. $\Phi(X+a)=\tau_{a} \Phi(X)$ for $X \subset Z^{v}$ and $a \in Z^{v}$

III. $\|\Phi\|=\sum_{X \ni 0}\|\Phi(X)\|<+\infty$

With respect to the norm introduced by condition III the interactions under consideration form a real Banach space $\mathscr{B}$. Two subsets of $\mathscr{B}$ will be of particular importance in the sequel. Firstly we define $\mathscr{B}_{1} \subset \mathscr{B}$ to be the set of interactions which involve only a finite number of particles i.e. those interactions which are such that $\Phi(X)=0$ for $N(X)$ sufficiently large, where $N(X)$ is the number of points in the set $X$. Secondly we define $\mathscr{B}_{0} \subset \mathscr{B}_{1}$ to be the set of finite range interactions i. e. those interactions $\Phi$ for which $\Phi^{(k)}\left(0, x_{2}, \ldots x_{k}\right)=0$ unless $\left\{x_{2}, \ldots x_{k}\right\} \subset \Lambda$ for some finite $\Lambda$.

If we consider a system of particles on the finite set $\Lambda$ the interaction energy of this svstem due to the interaction $\Phi$ is then defined by

$$
U_{\Phi}(\Lambda)=\sum_{X \subset A} \Phi(X)
$$

and clearly satisfies the stability condition

$$
\left\|U_{\Phi}(\Lambda)\right\| \leqq N(\Lambda)\|\Phi\| \text {. }
$$

\section{Time Translations as Automorphisms}

The aim of this section is to demonstrate under suitable conditions, that the limit

$$
\tau_{t}^{\Phi} A=\lim _{\Lambda \rightarrow \infty} e^{i t U_{\Phi}(\Lambda)} A e^{-i t\rangle_{\Phi}(\Lambda)}, A \in \mathfrak{A}, \quad t \in R .
$$

exists and defines a group of automorphisms $A \rightarrow \tau_{t}^{\Phi} A$ which will then be interpreted as the group of time translations of the spin system. tators

We begin by introducing the following notation for multiple commu-

and

$$
[A, B]^{(1)}=[A, B]
$$

$$
[A, B]^{(n)}=\left[A,[A, B]^{(n-1)}\right] \text { for } n>1 .
$$

We then have the following two lemmas

Lemma 1. If $A \in \mathfrak{A}\left(\Lambda_{1}\right), \Phi \in \mathscr{B}_{1}$ and $N_{\Phi}$ is such that $\Phi(X)=0$ if $N(X)>N_{\Phi}$ then

$$
\left\|\left[U_{\Phi}(\Lambda), A\right]^{(N)}\right\| \leqq 2^{N}\|\Phi\|^{N}\|A\|_{M=1}^{N}\left\{(M-1)\left(N_{\Phi}-1\right)+N\left(\Lambda_{1}\right)\right\} .
$$

Proof. We have, using local commutativity and the definitions of Sec. 2

$$
\left[U_{\Phi}(\Lambda), A\right]^{(N)}=\sum_{\substack{X_{1} \subset A \\ X_{1} \cap A_{1} \neq \emptyset}} \sum_{\substack{X_{2} \subset A \\ X_{2} \cap S_{1} \neq \emptyset}} \cdots \sum_{\substack{X_{N} \subset A \\ X_{N} \cap S_{N-1} \neq \emptyset}}\left[\Phi\left(X_{N}\right),\left[\ldots\left[\Phi\left(X_{1}\right), A\right]\right]\right]
$$


where we have introduced the notation

and

$$
S_{1}=X_{1} \cup \Lambda_{1}
$$

Therefore

$$
S_{k}=X_{k} \cup S_{k-1} \text { for } 1<k<N
$$

$$
\begin{aligned}
& \left\|\left[U_{\Phi}(\Lambda), A\right]^{(N)}\right\| \\
& \qquad 2 \sum_{\substack{X_{1} \subset \Lambda \\
X_{1} \cap \Lambda_{1} \neq \emptyset}} \cdots \sum_{\substack{X \subset A \\
X_{N} \cap S_{N-1} \neq \emptyset}}\left\|\Phi\left(X_{N}\right)\right\|\left\|\left[\Phi\left(X_{N-1}\right),\left[\ldots\left[\Phi\left(X_{1}\right), A\right]\right]\right]\right\| .
\end{aligned}
$$

But using

we find that

$$
N\left(S_{N-1}\right) \leqq(N-1)\left(N_{\Phi}-1\right)+N\left(\Lambda_{1}\right)
$$

$$
\begin{aligned}
& \left\|\left[U_{\Phi}(\Lambda), A\right]^{(N)}\right\| \leqq 2\left[(N-1)\left(N_{\Phi}-1\right)\right. \\
& \left.\quad+N\left(\Lambda_{1}\right)\right]\|\Phi\| \sum_{\substack{X_{1} \subset \Lambda \\
X_{1} \cap \Lambda_{1} \neq \emptyset}} \ldots \sum_{\substack{X_{N-1} \subset \Lambda \\
X_{N-1} \cap S_{N-2} \neq \emptyset}}\left\|\left[\Phi\left(X_{N-1}\right) \ldots\left[\Phi\left(X_{1}\right), A\right]\right]\right\|
\end{aligned}
$$

Repetition of this method of estimation leads directly to the desired result.

Lemma 2. If $A \in \mathfrak{A}\left(\Lambda_{1}\right), \Phi_{1}, \Phi_{2} \in \mathscr{B}_{1}$ and $N_{\Phi}$ is such that $\Phi_{1}(X)=0$ $=\Phi_{2}(X)$ if $N(X)>N_{\Phi}$ then

$$
\begin{aligned}
& \left\|\left[U_{\Phi_{1}}(\Lambda), A\right]^{(N)}-\left[U_{\Phi_{2}}(\Lambda), A\right]^{(N)}\right\| \\
& \quad \leqq N 2^{N}\left\|\Phi_{1}-\Phi_{2}\right\|\|\Phi\|^{N-1}\|A\| \prod_{M=1}^{N}\left\{(M-1)\left(N_{\Phi}-1\right)+N\left(\Lambda_{1}\right)\right\}
\end{aligned}
$$

where $\|\Phi\|=\max \left\{\left\|\Phi_{1}\right\|,\left\|\Phi_{2}\right\|\right\}$.

Proof. The proof of this lemma follows from noting the algebraic identity

$$
\begin{aligned}
{\left[U_{\Phi_{1}}(\Lambda), A\right]^{(N)} } & -\left[U_{\Phi_{2}}(\Lambda), A\right]^{(N)} \\
= & \sum_{M=0}^{N-1}\left[U_{\Phi_{1}}(\Lambda), \ldots\left[U_{\Phi_{1}-\Phi_{2}}(\Lambda),\left[U_{\Phi_{2}}(\Lambda), A\right]^{(M)}\right]\right]^{(N-M-1)}
\end{aligned}
$$

and then estimating the norm in the same manner as in Lemma 1 .

Using the above two lemmas we can readily derive the desired result. For $a_{i}>0, a_{i} \in Z(i=1,2, \ldots \nu)$ we introduce the notation

$$
\Lambda_{a}=\left\{x \in Z^{\nu} ;-a_{i} \leqq x_{i} \leqq a_{i}, i=1,2 \ldots \nu\right\} .
$$

Then we have

Theorem 1. If $\Phi \in \mathscr{B}_{1}, A \in \mathfrak{A}$ and $t \in R$ then the following limit exists in the norm topology

$$
\lim _{a_{1}, \ldots a_{\nu} \rightarrow \infty} e^{i t U_{\Phi}\left(\Lambda_{a}\right)} A e^{-i t U_{\Phi}\left(\Lambda_{a}\right)}=\tau_{t}^{\Phi} A
$$

and the mapping $A \rightarrow \tau_{t}^{\Phi} A$ defines a strongly continuous group of automorphisms of the $C^{*}$-algebra $\mathfrak{A}$ of quasi-local observables. 
Proof. Firstly we note that it is sufficient to prove the existence of the limit for strictly local $A$ as these elements are dense in $\mathfrak{A}$. Secondly we have for $\Phi$ of finite range and $2|t|\|\Phi\|\left(N_{\Phi}-1\right)<1$, due to lemma 1

$$
\begin{aligned}
\left\|e^{i t U_{\Phi}\left(A_{a}\right)} A e^{-i t U_{\Phi}\left(\Lambda_{a}\right)}-e^{i t U_{\Phi}\left(\Lambda_{b}\right)} A e^{-i t U_{\Phi}\left(\Lambda_{b}\right)}\right\| \\
\quad \leqq 2\|A\| \sum_{N>N(a, b)} \frac{2^{N}|t|^{N} \| \Phi \mid N}{N !} \prod_{M=0}^{N-1}\left\{M\left(N_{\Phi}-1\right)+N(\Lambda)\right\}
\end{aligned}
$$

where we have taken $A \in \mathfrak{A}(\Lambda)$ and $N(a, b)$ is an integer depending on the range of $\Phi$, and $a$ and $b . N(a, b)$ is an increasing function of $c_{i}=\min$ $\left(a_{i}, b_{i}\right) \quad(i=1, \ldots v)$. Due to the restriction $2|t|\|\Phi\|\left(N_{\Phi}-1\right)<1$ the series occurring in this estimate is convergent and thus we deduce the existence of the limit(1) for $\Phi$ of finite range and $2|t|\|\Phi\|\left(N_{\Phi}-1\right)<1$. To remove the first of these restrictions we note that due to lemma 2

$$
\begin{aligned}
& \left\|e^{i t U_{\Phi}\left(\Lambda_{a}\right)} A e^{-i t U_{\Phi}\left(\Lambda_{a}\right)}-e^{i t U_{\Phi_{1}}\left(\Lambda_{a}\right)} A e^{-i t U_{\Phi_{1}}\left(\Lambda_{a}\right)}\right\| \\
& \quad \leqq 2\|A\|\left\|\Phi-\Phi_{1}\right\| \sum_{N=1} \frac{\left.2^{N}|t|\right|^{N}\|\Phi\|^{N-1}}{(N-1 !)} \prod_{M=0}^{N-1}\left\{M\left(N_{\Phi}-1\right)+N(\Lambda)\right\}
\end{aligned}
$$

where we have introduced a second interaction $\Phi_{1} \in \mathscr{B}_{1}$ and assumed $\left\|\Phi_{1}\right\| \leqq\|\Phi\|$. Noting that this latter estimate is uniform in $a$ and that the series occurring on the right hand side is again convergent if $2|t|\|\Phi\|\left(N_{\Phi}-1\right)<1$ we may deduce the existence of the limit (1) for general $\Phi \in \mathscr{B}_{1}$ and small $t$. Explicitly for general $\Phi$ we choose a sequence $\left(\Phi_{n}\right)_{n \geqq 1}$ of finite range interactions which are such that $\left\|\Phi-\Phi_{n}\right\| \underset{n=\infty}{\longrightarrow} 0$ and $\|\Phi\| \geqq\left\|\Phi_{n}\right\|$ and then express (1) as a double limit. The above estimates justify the interchange of limits and the existence of the interchanged limit under the condition $2|t|\|\Phi\|\left(N_{\Phi}-1\right)<1$. Removal of this last restriction is however easy using the group property $e^{i\left(t_{1}+t_{2}\right) U_{\Phi}(\Lambda)} A e^{-i\left(t_{1}+t_{2}\right) U_{\Phi}(\Lambda)}=e^{i t_{1} U_{\Phi}(\Lambda)}\left\{e^{i t_{2} U_{\Phi}(\Lambda)} A e^{-i t_{2} U_{\Phi}(\Lambda)}\right\} e^{-i t_{1} U_{\Phi}(\Lambda)}$.

The strong continuity of the automorphisms $A \rightarrow \tau_{t}^{\Phi} A$ follows immediately from Lemma 2 and concludes the proof of the theorem.

One may remark at this point that the methods used above also allow us to deduce the existence of the mapping $A \rightarrow \tau_{t}^{\Phi} A$ for small imaginary values of $t$.

\section{Equilibrium States}

In I it was shown that for $\Phi \in \mathscr{B}$ the thermodynamic free energy $P(\Phi)$ of the spin system can be defined by

$$
P(\Phi)=\lim _{\Lambda \rightarrow \infty} \frac{1}{N(\Lambda)} \log \operatorname{Tr}_{\mathscr{H}_{\Lambda}}\left(e^{-U_{\Phi}(\Lambda)}\right)
$$

and the function $\Phi \rightarrow P(\Phi)$ is convex continuous. Further it was demonstrated that if $T \subset \mathscr{B}$ is the set of $\Phi$ for which the graph of $P(\cdot)$ has 
a unique tangent plane then, for $\Phi \in T$ and $A \in \mathfrak{A}\left(\Lambda_{1}\right)$ strictly local, the limit

$$
\varrho_{\Phi}(A)=\lim _{\Lambda \rightarrow \infty} \frac{1}{N(\Lambda)} \sum_{\substack{x \in \Lambda \\ A_{1}+x \subset \Lambda}} \operatorname{Tr}_{\mathscr{H}_{\Lambda}}\left(e^{-U_{\Phi}(\Lambda)} \tau_{x} A\right) / \operatorname{Tr}_{\mathscr{H}_{\Lambda}}\left(e^{-U_{\Phi}(\Lambda)}\right)
$$

exists and the $\varrho_{\Phi}(A)$ define a state over the $C^{*}$-algebra $\mathfrak{U}$ of (quasi-)local observables. The equilibrium state $\varrho_{\Phi}$ defined in this manner is invariant under space translations and is in fact an extremal element of the set of $Z^{v}$-invariant states over $\mathfrak{A}$. Physically this last statement means that for $\Phi \in T$ the system is in a single thermodynamic phase.

As time translations form a group of automorphisms of $\mathfrak{A}$ it is possible to derive certain properties of the equilibrium state with respect to these translations. Such properties have been considered by HAAG, HugenHOLTZ, and WrNNINK [2]; although we do not have quite the same input as these authors require we can derive the following theorem, and its corollary, as given in [2].

Theorem 2. If $\Phi \in T \cap \mathscr{B}_{1}$ then the equilibrium state $\varrho_{\Phi}$ has the following two properties

1. Invariance $\varrho_{\Phi}$ is invariant under time-translations i.e.

$$
\varrho_{\Phi}\left(\tau_{t}^{\Phi} A\right)=\varrho_{\tau}(A), A \in \mathfrak{A}, t \in R .
$$

2. KMS Boundary condition. For $A, B \in \mathfrak{X}$ the function $\varrho_{\Phi}\left(A\left(\tau_{t}^{\Phi} B\right)\right)$ of $t$ can be continued analytically in the strip $0<I m t<1$ and is continuous on the boundaries of this strip. Similarly $\varrho_{\Phi}\left(\left(\tau_{t}^{\Phi} B\right) A\right)$ is analytic in the open strip $0>I m t>-1$ and continuous on the boundaries. The boundary values of these analytic functions satisfy the condition

$$
\varrho_{\Phi}\left(A\left(\tau_{t+i}^{\Phi} B\right)\right)=\varrho_{\Phi}\left(\left(\tau_{t}^{\Phi} B\right) A\right) \quad t \text { real } .
$$

Proof. It is sufficient to prove the invariance property for strictly local $A$. If the space average were absent in the right hand side of (2) we would have the same starting point as [2] and the proof would then follow from this reference. To handle this space average we proceed as follows. As space translations form a group of automorphisms of $\mathfrak{A}$ and are hence norm-preserving we can for $\varepsilon>0$ choose an $a_{0}$ such that

$$
\left\|\tau_{x}\left(e^{i U_{\Phi}\left(\Lambda_{a}\right) t} A e^{-i U_{\Phi}\left(\Lambda_{a}\right) t}\right)-\tau_{t}^{\Phi} \tau_{x} A\right\|<\varepsilon
$$

for $a>a_{0}$ and for all $x \in Z^{\nu}$. However, if $x$ is restricted to take values in a set $\Lambda_{b} \subset Z^{v}$ then it is easily checked that we can choose $a_{0}$ such that

$$
\left\|e^{i t U_{\Phi}\left(\Lambda_{a+b}\right)}\left(\tau_{x} A\right) e^{-i t U_{\Phi}\left(\Lambda_{a+b}\right)}-\tau_{x}\left(e^{i t U_{\Phi}\left(\Lambda_{a}\right)} A e^{-i t U_{\Phi}\left(\Lambda_{a}\right)}\right)\right\|<\varepsilon
$$

for $a>a_{0}$ and for all $x \in Z^{\nu}$. Using this estimate, cyclicity of the trace, and definition (2), the invariance of $\varrho_{\Phi}$ follows, in the same manner as in [2], from a series of simple estimates. 
Let us begin the proof of the Kubo-Martin-Schwinger boundary condition by defining, for $A, B \in \mathfrak{A}\left(\Lambda_{1}\right)$, the analytic function $t \rightarrow F_{A ; \Phi}^{B} \cdot A(t)$ by

$$
\begin{aligned}
F_{A, \Phi}^{B, A}(t)= & \frac{1}{N(\Lambda)} \sum_{\substack{x \in \Lambda \\
\Lambda_{1}+x \subset \Lambda}} \\
& \cdot \operatorname{Tr}_{\mathscr{H}_{\Lambda}}\left(e^{-U_{\Phi}(\Lambda)} e^{i t U_{\Phi}(\Lambda)}\left(\tau_{x} B\right) e^{-i t U_{\Phi}(\Lambda)}\left(\tau_{x} A\right)\right) / \operatorname{Tr}_{\mathscr{H}_{\Lambda}}\left(e^{-U_{\Phi}(\Lambda)}\right)
\end{aligned}
$$

and noting that due to the cyclicity to the trace we have the identity

$$
F_{A, \Phi}^{B, A}(t)=F_{A, \Phi}^{A, B}(t+i) .
$$

Next, following the methods of [2], we introduce for each function $f \in \mathscr{D}$, the space of infinitely often differentiable functions with compact support, the transform

$$
f(z)=\int d \omega e^{i \omega z} \tilde{f}(\omega), z \in \mathbb{C} .
$$

Then, from (5) we find

$$
\int d t f(t) F_{A ; \Phi}^{B ; A}(t)=\int d t f(t-i) F_{A ; \Phi}^{A} ;(t) .
$$

Now given $\varepsilon>0$ we can choose $a_{0}$ such that the estimate (3) remains valid and such that

$$
\left\|\left\{\left(e^{i t U_{\Phi}\left(\Lambda_{a+b}\right)}\left(\tau_{x} A\right) e^{-i t U_{\Phi}\left(\Lambda_{a+b}\right)}\right)-\tau_{x}\left(e^{i t U_{\Phi}\left(\Lambda_{a}\right)} A e^{-i t U_{\Phi}\left(\Lambda_{a}\right)}\right)\right\} \tau_{x} B\right\|<\varepsilon
$$

for $a>a_{0}$ and for all $x \in \Lambda_{b}$. These estimates together with definition (2) allow us to deduce that

$$
\lim _{c_{1} \ldots c_{\nu} \rightarrow \infty} F_{A_{c}, \Phi}^{B . A}(t)=\varrho_{\Phi}\left(\left(\tau_{t}^{\Phi} B\right) A\right)
$$

and thus from (6) we find that

$$
\int d t f(t) \varrho_{\Phi}\left(\left(\tau_{t}^{\Phi} B\right) A\right)=\int d t f(t-i) \varrho_{\Phi}\left(A\left(\tau_{t}^{\Phi} B\right)\right)
$$

for $A, B$ strictly local, and hence by continuity for all $A, B \in \mathfrak{A}$. The stated analyticity properties follow from this relations as pointed out in [2]. The argument of [2] may be paraphrased as follows. The function $t \rightarrow \varrho_{\Phi}\left(\left(\tau_{t}^{\Phi} B\right) A\right)$ is continuous bounded and hence its Fourier transform is a tempered distribution whose growth at infinite is at worst polynomial. However from (7) we find

$$
\int d t e^{-i \omega t} \varrho_{\Phi}\left(\left(\tau_{t}^{\Phi} B\right) A\right)=e^{\omega} \int d t e^{-i \omega t} \varrho_{\Phi}\left(A\left(\tau_{t}^{\Phi} B\right)\right)
$$

and hence this Fourier transform must decrease as $e^{-\omega}$ at infinity. This directly implies the desired analyticity.

Corollary. If $A \in \mathfrak{A}$ is such that $\varrho_{\Phi}\left(A^{*} A\right)=0$ then if follows that $A=0$. Using the Schwartz inequality we find that $\varrho_{\Phi}(A * A)=0$ implies that $\varrho_{\Phi}\left(A^{*} \tau_{t}^{\Phi} A\right)=0$ but then from the boundary condition $\varrho_{\Phi}\left(\left(\tau_{t}^{\Phi} A\right) A^{*}\right)=0$ and in particular $\varrho_{\Phi}\left(A A^{*}\right)=0$. But the conditions $\varrho\left(A^{*} A\right)=0=\varrho_{\Phi}\left(A A^{*}\right)$ 
are only simultaneously possible if $A$ is contained in a two-sided ideal of $\mathfrak{A}$. As $\mathfrak{A}$ is simple the only two-sided ideals are trivial and thus $A=0$.

The above corollary yields a statement similar to the Reeh-Schlieder theorem [3] in relativistic field theory and it is perhaps instructive to compare the elements necessary to derive this statement both in its field theoretic and its statistical mechanical setting. Whilst in field theory the property originates from invariance, locality, and the spectrum condition, in statistical mechanics it is essentially a result of invariance, and the KMS-boundary condition. This indicates that the boundary condition plays somewhat the same role as the spectrum condition, a point which is useful to bear in mind in the subsequent analysis. HAAG, HugenHoltz, and WINNINK [2] have already derived some of the algebraic consequences of this condition which are independent of locality and invariance under space translations. In the following section we derive consequences of this condition coupled with these latter properties.

\section{Invariant States and the KMS Boundary Condition}

In this section we will work in a more general framework than that adopted in previous sections. We consider a $C^{*}$-algebra $\mathfrak{A}$ with identity, the group $G=R^{\nu_{1}} \times Z^{\nu_{2}}$, and a representation $\tau$ of $G$ as automorphisms of $\mathfrak{A}$ such that $\mathfrak{A}$ is asymptotically abelian with respect to $G$, the group of space translations i.e.

$$
\left\|\left[A, \tau_{g} B\right]\right\| \underset{|g|=\infty}{\longrightarrow} 0, A, B \in \mathfrak{A}
$$

Corresponding to time translations we consider a further representation $\tau^{\prime}$ of $T=R$ as automorphisms of $\mathfrak{A}$ which commute with $\tau$ and we assume all these automorphisms to be strongly continuous. We are specifically interested in states $\varrho$ over $\mathfrak{A}$ which are invariant under both $\tau$ and $\tau^{\prime}$, i.e. states such that

$$
\varrho\left(\tau_{g} \tau_{t}^{\prime} A\right)=\varrho(A), g \in G, t \in T \text { and } A \in \mathfrak{A} .
$$

and we recall that associated with each such state is a representation $\pi \varrho$ of acting on a Hilbert space $\mathscr{H}_{\varrho}$ with cyclic vector $\Omega_{\varrho}$ and unitary representations $U_{\varrho}$ and $V_{\varrho}$, of $G$ and $T$ respectively, such that

$$
\pi\left(\tau_{g} \tau_{t}^{\prime} A\right)=U_{\varrho}(g) V_{\varrho}(t) \pi_{\varrho}(A) V_{\varrho}^{-1}(t) U_{\varrho}^{-1}(g) \text { and } U_{\varrho}(g) V_{\varrho}(t) \Omega_{\varrho}=\Omega_{\varrho}
$$

for all $A \in \mathfrak{A}, g \in G$ and $t \in T$. We denote by $E_{\varrho}$ the projection on all $G$-invariant vectors in $\mathscr{H}_{\varrho}$, thus $E_{\varrho}$ is such that

$$
U_{\varrho}(g) E_{\varrho}=E_{\varrho}, g \in G \text {. }
$$

Lemma 3. If the state @ satisfies the KMS boundary condition then it follows that

$$
V_{Q}(t) E_{Q}=E_{Q}, t \in R .
$$


Proof. Denoting by $F_{Q}$ the spectral projector associated with the unitary operator $V_{\varrho}$ we find immediately from the KMS boundary condition and invariance that

$$
\begin{array}{r}
\left(\Omega_{\varrho},\left[\pi_{\varrho}(A), \pi_{\varrho}\left(\tau_{g} B\right)\right] \Omega_{\varrho}\right)=\left(\Omega_{\varrho}, \pi_{\varrho}(A) \pi\left(\tau_{g} B\right) \Omega_{\varrho}\right)-\left(\Omega_{\varrho}, \pi_{\varrho}(A) \pi\left(\tau_{i}^{\prime} \tau_{g} B\right) \Omega_{\varrho}\right) \\
=\left(\Omega_{\varrho}, \pi_{\varrho}(A)\left(1-\int d F_{\varrho}(\omega) e^{-\omega}\right) U_{\varrho}(g) \pi_{\varrho}(B) \Omega\right) \quad A, B \in \mathcal{A}, g \in G .
\end{array}
$$

Next taking mean values over $G$ we find that the left hand side of this equations gives zero due to asymptotic abelianness whilst the mean value of $U_{\varrho}$ is $E_{\varrho}$ thus

$$
\left(\Omega_{\varrho}, \pi\left(A_{\varrho}\right)\left(1-\int d F_{\varrho}(\omega) e^{-\omega}\right) E_{\varrho} \pi_{\varrho}(B) \Omega_{\varrho}\right)=0 .
$$

Finally using cyclicity of $\Omega_{o}$ for $\pi_{\varrho}$ we deduce straightforwardly that $F(\Delta) E_{0}=0$ if $0 \notin \Delta$ and $F(\{0\}) E_{\varrho}=F(\{0\})$ from which the quoted result immediately follows.

The above lemma leads us to the following result.

Theorem 3. If $\varrho$ is a state over an asymptotically abelian algebra which is invariant under both space and time translations and satisfies the KMS boundary condition with respect to the latter, then the unitary representative $V_{Q}$ of time translations is contained in the weak closure of the set $\left\{\pi_{Q}(\mathfrak{Q})\right.$, $\left.U_{e}(G)\right\}$ of operators, representing the algebra and the group of space-translations respectively.

Proof. To demonstrate that $V_{\varrho}(T) \in\left\{\pi_{\varrho}(\mathfrak{R}), U_{\varrho}(G)\right\}^{\prime \prime}$ it is necessary and sufficient to show that for each $C \in\left\{\pi_{\varrho}(\mathfrak{A}), U_{\varrho}(G)\right\}^{\prime}$

However

$$
\left[V_{e}(t), C\right]=0, t \in T \text {. }
$$

$$
\begin{aligned}
\left(\Omega_{\varrho}, \pi_{\varrho}(A) C V_{\varrho}(t) \pi(B) \Omega_{\varrho}\right) & =\left(\Omega_{\varrho}, \pi_{\varrho}(A) C \pi_{\varrho}\left(\tau_{t}^{\prime} B\right) \Omega\right) \\
& =\left(\Omega_{\varrho}, \pi_{\varrho}(A) V_{\varrho}(t) \pi_{\varrho}(B) V_{\varrho}^{-1}(t) C \Omega_{\varrho}\right)
\end{aligned}
$$

and

$$
C \Omega_{\varrho}=E_{\varrho} C \Omega_{\varrho}
$$

where we have twice used the fact that $C \in\left\{\pi_{\varrho}(\mathfrak{A}), U_{\varrho}(G)\right\}^{\prime}$. Now (8) follows from (9) and (10) and Lemma 3.

In [4] and [5] it was demonstrated that the asymptotic abelianness condition ensured that the set $\left\{\pi_{\varrho}, U_{\varrho}\right\}^{\prime}$ is abelian. Thus the result of the above theorem can be restated; time translations leave the centre of the covariance algebra generated by the pair $\left\{\pi_{e}, U_{e}\right\}$ pointwise invariant. This result has an analogue in relativistic field theory (see ARAKI [6]).

Due to the fact that the commutant $\left\{\pi_{\varrho}, U_{e}\right\}^{\prime}$ is abelian one can derive (see [4] and [5]) unique integral decompositions of states $\varrho$, invariant under space-translations, into ergodic states i.e. states extremal among the set of states invariant under space translations. These decompositions are physically interpretable as the separation of thermodynamic 
phases. From Lemma 3 and the structure of the integral decompositions it is easily inferrable that if $\varrho$ is invariant under time translations and satisfies the KMS boundary condition then the ergodic states, associated with $\varrho$ via the decomposition, are also invariant under time-translations. We now wish to demonstrate a stronger result. We will show that the ergodic states arising in this manner also satisfy the KMS boundary condition. To this end we need the following two Lemmas.

Lemma 4. Let @ be a $G$-invariant state over $\mathfrak{A}$ and assume $\mathfrak{A}$ to be asymptotically abelian with respect to $G$. If $C \in\left\{\pi_{\varrho}, U_{o}\right\}^{\prime}$ there exists an $A \in \mathfrak{A}$ such that $\pi_{\varrho}(A)$ approximates $C$ strongly and if $C$ is hermitian $A$ may be chosen to be hermitian.

Proof. This lemma is an immediate consequence of the fact that $\left\{\pi_{\varrho}, U_{\varrho}\right\}^{\prime} \subset \pi_{\varrho}^{\prime \prime}$ (see [4]).

Lemma 5. Let $\varrho$ be a G-invariant state over a $C^{*}$-algebra $\mathfrak{A}$ which is asym ptotically abelian with respect to G. Further assume that $\varrho$ is invariant under time translations and satisfies the KMS boundary condition. If $C \in\left\{\pi_{\varrho}, U_{\varrho}\right\}^{\prime}, C=C^{*}$ and $\left\|C \Omega_{\varrho}\right\|^{2}=1$ then the state $\varrho_{C}$ over defined by

$$
\varrho_{C}(A)=\left(\Omega_{\varrho}, \pi_{\varrho}(A) C^{2} \Omega_{\varrho}\right)
$$

is also invariant under time translations and satisfies the KMS boundary condition.

Proof. The fact that $\varrho_{C}$ is invariant under time translations follows from $C^{2} \Omega_{\varrho}=E_{\varrho} C^{2} \Omega_{\varrho}$ and lemma 3 . To prove that $\varrho_{C}$ satisfies the KMS boundary condition we use lemma 4 and choose for $A \in \mathfrak{A}, A_{C} \in \mathfrak{A}$ such that $A_{C}=A_{C}^{*}$ and

$$
\left\|\left(C^{2}-\pi_{\varrho}\left(A_{C}\right)\right) \Omega_{\varrho}\right\|<\varepsilon,\left\|\left(C^{2}-\pi_{\varrho}\left(A_{C}\right)\right) \pi_{\varrho}\left(A^{*}\right) \Omega_{\varrho}\right\|<e .
$$

Now as $\varrho$ satisfies the KMS boundary condition was have for $f \in \mathscr{D}$

$$
\begin{aligned}
\int d t f(t)\left(\Omega_{\varrho}, \pi_{\varrho}(B) V_{\varrho}(-t) \pi_{\varrho}\left(A A_{C}\right) \Omega\right) & \\
& =\int d t f(t-i)\left(\Omega_{\varrho}, \pi_{\varrho}\left(A A_{C}\right) V_{\varrho}(t) \pi_{\varrho}(B) \Omega_{\varrho}\right) .
\end{aligned}
$$

But from (11) it then immediately follows that

$$
\int d t f(t) \varrho_{C}\left(\left(\tau_{t}^{\prime} B\right) A\right)=\int d t f(t-i) \varrho_{C}\left(A\left(\tau_{t}^{\prime} B\right)\right)
$$

however this latter condition is equivalent to the KMS boundary condition.

Theorem 4. Let $\varrho$ be a state over a separable $C^{*}$-algebra $\mathfrak{A}$, which is asymptotically abelian with respect to the group $G$ of space translation. Assume that $\varrho$ is invariant with respect to both $G$ and the group $T$ of time translations and that $\varrho$ satisfies the KMS boundary condition. It follows that there exists a unique integral decomposition of @ into extremal G-invariant states which are also T-invariant and satisfy the KMS boundary condition. 
The existence of a unique decomposition of a $G$-invariant state, over an asymptotically abelian algebra, into extremal $G$-invariant states is demonstrated in [4] and [5]. In [4] it is shown that this decomposition can be obtained from the unique central decomposition of the covariance algebra generated by the pair $\left\{\pi_{\varrho}, U_{\varrho}\right\}$ (the separability assumption for $\mathfrak{A}$ ensures the existence of the central decomposition). The fact that the states occurring in this decomposition are invariant under time-translations and satisfy the KMS boundary condition follows directly from the theory of the central decomposition and the preceding lemma.

It might be remarked that the existence and uniqueness of the integral decompositions arising in the above theorem are derived in [5] under rather weak separability conditions of a physical origin. We have assumed the separability of $\mathfrak{A}$ in the above theorem for conciseness but the theorem should be valid even in the more general framework of [5]. Secondly we note that some extremal $G$-invariant states have non-trival decompositions into states which are invariant under a closed subgroup of $G$ (see [4] and [7] for details). It follows that these latter decompositions, which correspond to the spontaneous breakdown of translation symmetry, also respect the property of invariance under time-translations and the KMS boundary condition.

Finally it is of interest to examine the spectral properties of the unitary operators $U_{\varrho}$ and $V_{\varrho}$ which implement space and time translations respectively. Such properties have already been extensively studied for states invariant under space translations (see [4] and [7]).

Theorem 5. Let $\varrho$ be a state over a $C *$-algebra $\mathfrak{A}$, which is asymptotically abelian with respect to the group $G$ of space translations, and assume $Q$ is an extremal G-invariant state which is also invariant under the group $T$ of time translations and satisfies the $K M S$ boundary condition. Let $E$ and $F$ be the projection-valued measures associated with the unitary operators $U_{\varrho}(G)$ and $V_{\varrho}(T)$, which implement space and time translations respectively, via the spectral representations

$$
U_{\varrho}(g)=\int d E(p) e^{+i p \cdot g} \text { and } V_{\varrho}(l)=\int d F(\omega) e^{+i \omega t} .
$$

Then 1. $\operatorname{supp} E=-\operatorname{supp} E ; \operatorname{supp} E+\operatorname{supp} E \subset \operatorname{supp} E$

2. $\operatorname{supp} F=-\operatorname{supp} F ; \operatorname{supp} F+\operatorname{supp} F \subset \operatorname{supp} F$

3. if $S_{d}^{E}=\{p ; E(\{p\}) \neq 0\}$ then $S_{d}^{E}=-S_{d}^{E} ; S_{d}^{E}+S_{d}^{E} \subset S_{d}^{E}$

and if $p \in S_{d}^{E}$ then $E(\{p\})$ is one dimensional

4. each discrete eigenstate of $U_{\varrho}$ is invariant under $V_{\varrho}$ i.e.

$$
V_{\varrho}(t) E(\{p\})=E(\{p\}) \text {. }
$$

Proof. Property 3 is proved in [4]. The additivity of supp $E$ is demonstrated in [7] and, noting that space and time translations commute, the proof easily extends to show that supp $F$ is additive. The symmetry 
of supp $E$ and supp $F$ follow immediately from the KMS boundary condition in the form

$\left(\Omega_{\varrho}, \pi_{\varrho}(A) E(-p) F(\omega) \pi_{\varrho}(B) \Omega_{\varrho}\right)=\left(\Omega_{\varrho}, \pi_{\varrho}(B) E(p) F(-\omega) \pi_{\varrho}(A) \Omega_{\varrho}\right) e^{\omega}$

(to be understood in the sense of distributions). Finally property 4 follows from Lemma 3 if we apply this lemma to a subgroup of $G$ chosen such that $E(\{p\})$ is invariant under the subgroup of translations.

Note that all statements of the theorem except the additivity properties are valid without the extremality assumption for $\varrho$. It is possible that a more detailed investigation of the KMS boundary condition will yield further information concerning the discrete spectrum of time translations and its link with the discrete spectrum of space translations.

Acknowledgements. The author is indebted to S. Grashow for his hospitality at Harvard where the major part of this work was carried out. During initial stages of the work a number of helpful discussions with V. GLASER are acknowledged.

\section{References}

1. Robinson, D. W.: Commun. Math. Phys. 6 (1967).

2. HAaG, R., N. Hugenholtz, and M. Winnink: Commun. Math. Phys. 5, 215 (1967).

3. ReeH, H., and S. Scrilieder: Nuovo Cimento 22, 1051 (1961).

4. Kastler, D., and D. W. Robinson: Commun. Math. Phys. 3, 151 (1966).

5. Ruelle, D.: Commun. Math. Phys. 3, 133 (1966).

6. Arakr, H.: Progr. Theoret. Phys. (Kyoto) 32, 844 (1967).

7. Robinson, D. W., and D. Ruelle: Ann Inst. Henri Poincare VI, 299 (1967).

8. Glimu, J.: Trans. Am. Math. Soc. 95, 318 (1960).

Dr. D. W. Robinson

Theoretical Physics Division CERN

Genf, Schweiz 